AUR Leishmania

TbAUR1 1 T. brucei

TCAUK1-T. cruzi

AUR $S$. Cerevisiâ MOR---1

AUK $\bar{B} \_$. pombe MS-----

AURA- $H$. Sapiens MDRSKENCISGPVKATAPVGGPKRVVTQQFPCQNPLPVNSGQAQRVLCPSNS--SQRIP

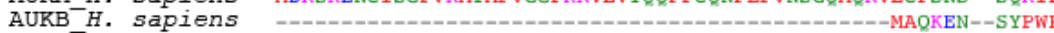

AUR Leishmania -

TbAUR1_T. brucei --

TCAUK1-T. Cruzi ---

AUR $S$. cerevisiae IS---------HSPQQRNPNSKIPSPVREKLNRLPVNNKKFLDMESSKIPSPIRKA

AUKB $S$. pombe PGRQQLLR----LAVSNQRQVNNVS-------LANGKEN----KRTSNSKFNSSLRKIE

AUKA ${ }^{-} H$. Sapiens LOAOKLVSSHKPVONOKOKOLOATSVPHPVSRPLNNTOKS----KOPLPSA--PENNPEE

AURB_H. sapiens YGRQTAPSGLS---TLPQRVLRKEPVT-PSAL-VLMSRSN----V-----Q--PTAAPGQ

\title{
ACTIVE SITE
}

AUR Leishmania NVVNNFIITPSSPKSEWTI HDFELLHKLGGGNYGDVYLASVRKSNYVVAIKKLSIKKLAE TbAUR1 $T$. bruce $i$ RVVEDF IVLPPTPKSKVKLSDFELLHKLGGGNYGDVHLASVKDCNFVCALKRLSIKKLAD TCAUK $1-T$. CrUzi OVVASYIALPKAPRSDWKASDFEMLHKLGGGNYGDVYLASVRDCNFVCAIKKLSIKKLAE AUK $S$. cerevisiae SSKMI HENKKLPKFKSLSLDDFELGKKLGKGKFGKVYCVRHRSTGY ICALKVMEKEEIIK AUK $\bar{B}$ S. pombe EPIAGVPSAGPQWREFHIGMFEIGKPLGKGKFGRVYLAKEKKTGF IVALKTLHKSELVD AUKA ${ }^{-} H$. sapiens -ELASKQKNEESKKRQWALEDFEIGRPLGKGKFGNVYLAREKQSKF ILALKVLFKAQLEK AUKB $^{-} H$. sapiens KVMENSSGTPDILTRHFTIDDFE I GRPLGKGKF GNVYLAREKKSHF IVALKVLFKSOIEK

$$
\text { **: : ***:***: : : : * *: : }
$$
ACTIVE SITE

AUR Leishmania FDIVNOLRREIEIAFNTR HKYLLRTYAYFFDEHDIYLILEPCSNGMLYSELNRVKLFPPP TbAUR1 T. brucei FDIATOLRREIEIAFNTRHKYLLRTYAYFFDETDIYLIMEPCSNGMLYTELNRVKCFAPP TCAUK ${ }^{-}$T. crUzi FDIVAOLRREIEIAFHTRHRYLLRTYGYFFDDTDIYLILEPCSNGMLYTELNRVKCFPPP AUR $S$. cerevisiae YNLOKOFRREVEIOTSLNHPNLTKSYGYFHDEKRVYLLMEYLVNGEMYKLLRLHGPFNDI AUK $\bar{B} S$. pombe SKIEKOVRREIEIOSNLRHKN ILRLYGHFHDEKRIYLILEF AGRGELYOHLRRAKRFSEE AURA $^{-} H$. Sapiens AGVEHOLRREVEIOSHLRHPNILRLYGYFHDATRVYLILEYAPLGTVYRELOKLSKFDEO

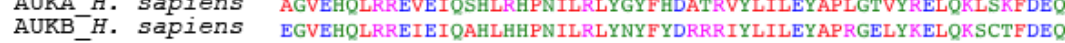

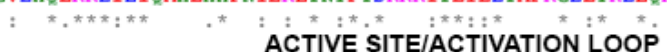

AUR Leishmania TbAUK1 T.brucei TCAUK1-T. Cruzi

AUR S. cerevisia

AUK $\bar{B}$ S. pombe

AUKA H. sapiens

AUKB H. sapiens

TAARYVAOLAEALLYLHOHHILHRDIKPENILLDHHONIKLADFGWSVH-DPLNRRKTSC TAARYVAOLAEALLYLHOHHILHRDIKPENILLDHNNNI KLADFGWSVH-DPDNRRKTSC TAARYVAQLAEALLYLHOHHILHRDI KPENI LLDHNQNIKLADFGWSVH-DPHNRRKTSC LASDY I YQIANALDYMHKKNI IHRDI KPENI LIGFNNVI KLTDF GWS I INPPENRRKTVC VASKY I FOMANALSYLHKKHVIHRDI KPENILLGIDGE I KLSDFGWSVH-APSNRRTTLC RTATYITELANALSYCHSKRVIHRDIKPENLLLGSAGELKIADFGWSVH-APSSRRTTLC RTATIMEELADALMYCHGKKVIHRDIKPENLLLGLKGELKIADFGWSVH-APSLRRKTMC $:: \quad::: * ; * * * *:,:: * * * * * * *: *: \quad: *:: * * * * *: \quad * * * * * *$

AUR Leishmania Y brucei GTPEYFPPEIVGRQAYDTSADLWCLGIFCYELLVGKTPFVGK-DTDQICKNIHSMHFKIP CAUK1 T. CIUZi GTPEYFPPEIVGRQPYDTSADLWCLGIFCYELLVGKTPFVSK-DTENICKKIHAMOYTIP AUR $s$. cerevisiae GTIDYLSPEMVESREYDHTIDAWALGVLAFELLTGAPPFEEE-MKDTTYKRIAALDIKMP AUK $\bar{B} S$. pombe GTLDYLPPEMVEGKEHTEKVDLWSLGVLTYEFLVGAPPFEDMSGHSATYKRIAKVDLKIP AURA $^{-} H$. Sapiens GTLDYLPPEMIEGRMHDEKVDLWSLGVLCYEFLVGKPPFEAN-TYQETYKRISRVEFTFP AUKB_H. sapiens GTLDYLPPEMIEGRMHNEKVDLWCIGVLCYELLVGNPPFESA-SHNETYRRIVKVDLKFP

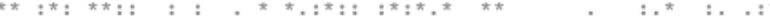

AUR Leishmania DSVPPEAKDLISNLLIREGSKRLALHRVLSHPFLLKYYYVPNGITPPTGKRPRS----TbAUR1 T. brUcei DNIPSEAKDLIANLLLRDGSRRLALHRVVNHOFLLKYYYLPNNLOPPTGKRPRLDAEPTA

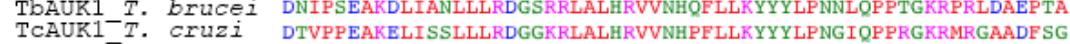
AUR S. Cerevisiaes AUKB. $\bar{B}$. pombe

AURA $H$. sapiens AUKB - . sapiens SNISQDAQDLI LKLLKYDP KDRMRLGDVKMHPWI LRNKPFWENKRI SFVPPDARDLI SRLLOHNPEKRMSLEOVMRHPWIVKYKDSWTRKSSESS---------DFVTEGARDLISRLLKHNPSORPMLREVLEHPWITANSSKPSNCONKESASKOS----ASVPTGAQDLISKLLRHNP SERLPLAQVSAHPWVRANSRRVLPP SALQSVA---------

AUR Leishmania

Figure S4: Multiple sequence alignment of Aurora Kinase (AUK) orthologous from the indicated species. Sequences were aligned using the Clustal Omega tool (https://www.ebi.ac.uk/Tools/msa/clustalo/). The active site and the activation loop of the enzyme from those species is indicated. 